\title{
Improving the Precision of Dynamic Forest Parameter Estimates Using Landsat
}

\author{
Evan B. Brooks ${ }^{a}$, John W. Coulston ${ }^{b}$, Randolph H. Wynne ${ }^{a}$, and Valerie A. Thomas ${ }^{a}$ \\ aDepartment of Forest Resources and Environmental Conservation, Virginia Tech, Blacksburg, VA, USA \\ bUSDA Forest Service Southern Research Station, Forest Inventory and Analysis Unit, Knoxville, TN, USA
}

\section{Abstract}

The use of satellite-derived classification maps to improve post-stratified forest parameter estimates is well established. When reducing the variance of post-stratification estimates for forest change parameters such as forest growth, it is logical to use a change-related strata map. At the stand level, a time series of Landsat images is ideally suited for producing such a map. In this study, we generate strata maps based on trajectories of Landsat Thematic Mapper-based normalized difference vegetation index values, with a focus on post-disturbance recovery and recent measurements. These trajectories, from 1985-2010, are converted to harmonic regression coefficient estimates and classified according to a hierarchical clustering algorithm from a training sample. The resulting strata maps are then used in conjunction with measured plots to estimate forest status and change parameters in an Alabama, USA study area. These estimates and the variance of the estimates are then used to calculate the estimated relative efficiencies of the post-stratified estimates. Estimated relative efficiencies around or above 1.2 were observed for total growth, total mortality, and total removals, with different strata maps being more effective for each. Possible avenues for improvement of the approach include the following: (1) enlarging the study area and (2) using the Landsat images closest to the time of measurement for each plot. Multitemporal satellite-derived strata maps show promise for improving the precision of change parameter estimates.

Keywords: harmonic regression, hierarchical cluster analysis, FIA, site index, post-stratification

\section{Introduction}

The use of satellite and other remote sensing data to supplement in situ measurements of forest variables is a well-established practice (Hansen et al., 2009; Fransson, 2000; Katila and Tomppo, 2002; McRoberts and Hansen, 1999; Nilsson et al., 2005; Wynne et al., 2000). There are many advantages to 
the use of satellite data, among them being increased coverage, reduced cost, and more frequent (and regular) measurement. These advantages make such data useful in assisting the scale-up of field observations to estimate population biophysical parameters of forests.

These parameters may be categorized into two groups. Status parameters define the condition of the forest at a point in time. Examples include total carbon stock, average canopy cover, and total species diversity. In contrast, change parameters define the manner in which the forest changes over time. Examples of these include total forest growth, total removals, total mortality, and total carbon flux.

The Forest Inventory and Analysis (FIA) program of the USDA Forest Service uses satellite data to augment its field measurements (McRoberts et al., 2002a; McRoberts et al., 2006; McRoberts, 2010). By assigning the field plots to image-derived strata, the FIA program is able to obtain more precise estimates of forest parameters than would have otherwise been possible or financially feasible.

\subsection{Post-stratification}

Stratified sampling and post-stratification (PS) are standard methods for increasing the precision of estimates given a fixed sample size (Cochran, 1977). In stratified sampling, a population is divided into mutually exclusive strata, and within each stratum a random sample is taken. It is assumed that the strata represent distinct subsets of the population which are sufficiently different as to warrant the separate treatment. An example would be estimating a parameter based on the land cover type, in which case one would stratify along classes of land cover based on remote sensing data. The sample size of strata may be based on either proportionate allocation or disproportionate (optimum) allocation, the latter case being desirable when additional sampling is needed in strata exhibiting greater variability. In either case, one would expect increased precision from using stratified designs instead of a simple random sample (SRS) design (Cochran, 1977).

In the case of PS, the sample is not defined based on the strata. Instead, the sample is assigned to strata which have been generated via some other approach (often from ancillary data, as is the case in this study). When the strata effectively partition the sample according to the measured variables of interest,

55 this approach can be nearly as precise as proportional stratified sampling (Cochran, 1977).

\subsection{Post-stratification and the FIA program}

57 PS estimation is used by the FIA program (Bickford, 1952; Chojnacky, 1998; LaBau et al., 2007;

58 McRoberts et al., 2012; Scott et al., 2005; Westfall et al., 2011). When considering continuous forest 
change over time. The FIA has used PS, starting with aerial imagery, since 1952 (Bickford, 1952). Because of the cost, time and effort required to obtain and interpret aerial images and the relative temporal sparseness of such images, the FIA program transitioned to primarily using digital and satellite imagery around the turn of the millennium (Hansen and Wendt, 2000; McRoberts et al., 2002a). In the decade that followed, researchers enjoyed success in cheaply improving the precision of many status forest parameter estimates using Landsat and its products (Hansen and Wendt, 2000; Hoppus and Lister, 2003; McRoberts et al., 2002b; McRoberts, 2010; Musy et al., 2006; Wayman et al., 2001; Wynne et al., 2000), such as the National Land Cover Dataset (Fry et al., 2006). The FIA program currently uses Landsat-based stratification methods to increase the precision of estimates for status parameters such as forest area and forest volume. One such method stratifies plots according to the number of forested pixels in a $5 \times 5$ neighborhood, resulting in a variety of strata based on the density of forested pixels around the target pixel (Hoppus and Lister 2003). McRoberts et al. (2002a) developed another approach that defines four strata of forest, forested edge, nonforested edge, and nonforest.

While there has been substantial work done with PS for status parameter estimates, published work has suggested that methods which are effective for status parameter estimation are less so for change parameter estimation (McRoberts et al., 2006). There is relatively little in the literature with regards to change parameter estimation. To develop strata maps related to forest growth from satellite data, multiple images through time are needed. Strata may be based on the time and severity of change or on the pattern of regeneration after a disturbance. Methods such as the vegetation change tracker (Huang et al, 2010), LandTrendr (Kennedy et al., 2010), exponentially weighted moving average change detection (Brooks et al., 2014), and the continuous monitoring of forest disturbance algorithm (Zhu et al., 2012) can produce stratification criteria for time and severity of disturbance, but they may not be able to provide sufficient regrowth information by themselves. Accordingly, the primary objective of this study was to test the effectiveness of using a Landsat-derived time series in PS estimation of forest area, total carbon stock, area of planting, area of cutting, total growth, total mortality, and total removals.

\section{Data}

The study area for this work was in west-central Alabama, USA (Fig. 1). The area is largely forested, and the dominant forest type is loblolly pine (Pinus taeda). 


\subsection{FlA plot data}

90 We used FIA phase 2 plot data corresponding to the study area described above. The resulting 977 plots

91 covered all land uses and ownerships.

92 The FIA sample locations are approximately systematic and are assumed to produce an equal probability sample (McRoberts et al., 2006). GPS positional accuracy of plot locations is approximately 8 to 20 meters (McRoberts, 2010). The sample for this study is divided into seven rotating panels, giving a seven-year remeasurement period for a set of plots with remeasurements for this study covering the years 2004-2010.

The FIA phase 2 plot design is detailed in Bechtold and Scott (2005). The plot consists of four circular subplots of radius 7.3 meters ( $24 \mathrm{feet}$ ) each, arranged with one subplot in the center and the remaining three equidistant from the central subplot at a distance of 36.6 meters ( 120 feet) with azimuths of 0 , 120 , and 240 degrees. The total extent of the four subplots is about $675 \mathrm{~m}^{2}$ (1/6 acre). For each plot land use is determined, and for those plots determined to contain a forest land use (in whole or in part) additional forest measurements are taken, including but not limited to tree diameters, tree heights, tree

103 species, and forest type (Burkman, 2005a; Burkman, 2005b).

\subsection{Satellite data}

105 We used Landsat 5 Thematic Mapper data (L1T) from WRS-2 path 21 row 37 covering our study area

106 (Fig. 1) from 1985-2011, downloaded from USGS. The temporal distribution of the images is shown in

107 Fig. 2. Note that while any given year may lack an image for a particular season, the temporal coverage

108 improves for any three year period. For simplicity, we used only Landsat 5 as a data source, choosing 109 only scenes with $10 \%$ or less nominal cloud cover to reduce incoming noise in the time series. The 110 resulting stack comprised 174 images in total. We further processed the images through the LEDAPS 111 algorithm (Masek et al., 2006) to convert them to surface reflectance, and we also used a band112 minimum based dark object subtraction (Chavez, 1988) with water bodies in the study area as the 113 calibration objects.

114 After preprocessing, we computed normalized difference vegetation index values (NDVI) (Tucker, 1979)

115 for each image and stacked the resulting images in date order. We also generated a stack of squared 116 NDVI values because of its relationship to fractional vegetation cover (Choudhury et al. 1994). NDVI, 117 squared NDVI, and image acquisition dates were used to generate strata maps. 
119 This study consists of three components: generation of a strata map from Landsat-based temporal

120 trajectories, PS estimation of parameters based on the strata map, and comparison of the variance of

121 the post-stratified estimates to the variance of estimates using the SRS estimator.

122

123

\subsection{Strata map generation}

We generated strata maps by deriving temporal trajectories from the Landsat data, refining them into objects for classification, and classifying these objects via a hybrid clustering approach.

\subsubsection{Trajectory generation}

We used harmonic regression (HR) (Brooks et al., 2012; Brooks et al., 2014) to compute trajectories of NDVI by year over the study timeframe. A brief summary of the use of HR for this study follows; for full details on the method, we suggest referring to Brooks et al. (2014).

For a given year, we took all acquired images from that year and both the years preceding it and succeeding it. Corresponding acquisition day numbers were scaled for convenience from $[1,365]$ to $[0,2 \pi]$, and denoted as $\underline{T}$. From this, we created a design matrix consisting of a column of 1 's and columns corresponding to sines and cosines of $\underline{T}$ for two harmonics, previously shown to capture the majority of the seasonal variability in the time series (Brooks et al., 2014; Moody and Johnson, 2001; Roerink et al., 2000).

For each pixel in the image, we used this design matrix in conjunction with the associated NDVI time series to estimate harmonic coefficients via least squares estimation. To remove short-duration anomalies in the time series, we calculated residuals based on initial coefficient estimates and, for that pixel, omitted any dates with unusually large residuals (defined as 2 standard deviations away from 0 ) before recalculating the coefficient estimates from the remaining data. We retained only the constant coefficient estimate thus obtained, treating this value as the representative value for that pixel for that year.

We performed the above process for each available year: 1986-2010, resulting in a time series trajectory of 25 temporally auto-correlated values, repeating the process on the squared NDVI stack to approximate trajectories of fractional vegetation cover. Fig. 3 shows trajectories of NDVI-based harmonic coefficient estimates from three example pixels. All three pixels are in loblolly pine (Pinus taeda) stands, and all three show some level of disturbance over their histories, although the degree and timing of the disturbances varies. 


\subsubsection{Cluster object generation}

149 The criteria of minimal heterogeneity within strata and maximal heterogeneity between strata are the same criteria underpinning cluster analysis. Thus, a cluster analysis will produce useful strata maps, provided that appropriate clustering object (CO) types are used. In this study, the chosen CO type should

152 attempt to capture change through time. Based on our interest in assessing site quality by tracking regrowth rates after disturbance and the recognition that the FIA plot data used in the study were acquired relatively recently, we explored two avenues with respect to $\mathrm{CO}$ selection.

\subsubsection{Clustering objects based on minimum value}

Consider the case of mimicking site index first, by noting that rapid regrowth of an area suggests a

157 higher site index. In general, a forest stand grown from planting will grow most quickly in the first few 158 years after planting, as evidenced by the rapid increase in leaf area index (LAI) in these first years

159 (Sampson et al., 2008). Recognizing this, the first CO type in this study estimates the point in time at which the forest in a pixel is disturbed and includes a set number of subsequent observations from that point.

162 We used a simple method of estimation for disturbance timing, taking the year for which the greatest decrease in NDVI was measured to be the year of disturbance. We took the subset of the trajectory 164 including this year and the seven subsequent years as the CO. Disturbances in the last seven years of 165 the time series were excluded to ensure seven years' worth of recorded data after the disturbance. 166 This CO type, denoted throughout this work as the post-minimum type, is depicted in Fig. 4a, showing 167 the extracted sub-trajectories for the example pixels from Fig. 3.

\subsubsection{Clustering objects based on recent measurements}

169 We took NDVI trajectories corresponding to the years 2002-2009, recognizing that this timeframe would 170 be comparable with the specific 7-year intervals from the plot measurements, even if those plots were 171 not measured in 2002 and 2009. This CO type, denoted as the 2002-2009 type, is depicted in Fig. 4b.

172 We also took a relative approach as follows. For the 2002-2009 period, we took the squared NDVI 173 trajectory (not to be confused with the square of the mean-coefficient NDVI trajectory used in the other 174 CO generations) as a proxy for fractional vegetation cover, used the 2002 value as a baseline reference, 175 and calculated relative change values for 2003-2009 by differencing. This CO type, denoted as modified 176 2002-2009, is depicted in Fig. 4c. 


\subsubsection{Classification}

178 We used each collection of COs as the inputs in an algorithm based on hierarchical cluster analysis (HCA)

179 and nearest-neighbor assignment (Tomppo et al., 2008), as demonstrated in Fig. 5. We chose HCA

180 because it allowed us to sequentially explore the changes in the strata maps as the number of strata

181 increased: in moving from an $n$-cluster solution to an $(n+1)$-cluster solution, only the least coherent

182 cluster of the $n$-cluster solution is broken up; all other assignments remain stable.

From the collection of all trajectories, we chose a training sample (Fig. 5a). This sample may be randomly generated or chosen to capture expected variation in the trajectories (e.g., drawing pixels from known forest regions of interest). For our sample, we created a distance matrix using the Manhattan, or taxicab, distance, which sums the absolute values of the differences between the trajectories at each point along those trajectories. We chose this distance metric over the Euclidean metric because it requires no multiplications, making it computationally more efficient, and it tends not to penalize single-value aberrations as heavily because deviations are summed without squaring.

Once the distance matrix was created, we performed HCA on the sample to generate solution sets of classes (Fig. 5b), identifiable with strata in this study. HCA sequentially agglomerates the trajectories into clusters using a linkage method which calculates the distances between all clusters at each step and links the two clusters with the minimum calculated distance. We used Ward's method (Ward, 1963) for our linkage method. Because the agglomeration is sequential, we needed to run the algorithm only once to generate multiple cluster solution sets.

Once the sample classification was completed, we classified the remaining trajectories by a nearestneighbor approach to the calculated cluster centroids, again using the Manhattan distance to compute between-trajectories distances (Fig. 5c and d). We repeated this process for each desired cluster solution set. Since the trajectories are identifiable with pixels in the study area, the final product of this process is a multilayered raster wherein each layer depicts a different strata map for the specified cluster solution sets.

\subsection{Post-stratification and estimation}

The estimators that follow in this section are based on those of Scott et al. (2005). As a general rule, we made the population estimates by estimating stratum means per unit area from the observations (FIA plots, assigned to strata based on the pixels containing the plot centers) and multiplying these means by the corresponding stratum areas. Note as well that the FIA program recommends a minimum of 10 
plots per stratum for stability in the parameter estimates (Westfall et al., 2011). This effectively limits

208 the number of strata available.

209 From our study area with total area $A$, we used $n$ observations of a variable $y$. The $n$ observations were

210 from FIA permanent plots. We then took an independently derived collection of $H$ strata forming a

211 partition of the study area (derived in our case from Landsat data) so that the $h^{\text {th }}$ stratum contains $n_{h}$

212 of the $n$ observations, denoting the observations in the $h^{\text {th }}$ stratum as $y_{1 h}, y_{2 h}, \ldots, y_{n_{h} h}$. In an ideal

213 situation, the stratification would generate a homogenous grouping of observations within each stratum

214 and observations would be heterogeneous among strata.

215 We defined $w_{h}$, the weight of the $h^{\text {th }}$ stratum, as the proportion of the study area assigned to that 216 stratum, and calculated it accordingly (in our case, the number of pixels in the $h^{\text {th }}$ stratum divided by

217 the number of pixels in the entire study area). We treated the weights as known quantities since a wall-

218 to-wall strata map of the study area is used. Then we obtained the total PS estimate $\left(\hat{y}_{s t}\right)$ and its

219 estimated variance $\left(\operatorname{Var}\left(\hat{y}_{s t}\right)\right)$,

$$
\hat{y}_{s t}=A \sum_{h=1}^{H} w_{h} \bar{y}_{h}
$$

221 and

$$
\operatorname{Var}\left(\hat{y}_{s t}\right)=\frac{A^{2}}{n}\left[\sum_{h=1}^{H} w_{h} n_{h} \operatorname{Var}\left(\bar{y}_{h}\right)+\sum_{h=1}^{H}\left(1-w_{h}\right) \frac{n_{h}}{n} \operatorname{Var}\left(\bar{y}_{h}\right)\right]
$$

223 respectively, where $\bar{y}_{h}$ is the mean of the observations from the $h^{\text {th }}$ stratum and $\operatorname{Var}\left(\bar{y}_{h}\right)$ is the

224 variance of that within-stratum mean. Completely non-sampled plots were excluded from the analysis 225 and were assumed to be missing at random within strata (Patterson et al. 2012). Partially non-sampled 226 plots were handled according to Scott et al. (2005).

227 We used the estimated relative efficiency (ERE) (Eq. 3) to quantify the gains in precision from the 228 estimator:

$$
E R E=\frac{\operatorname{Var}\left(\hat{y}_{S R S}\right)}{\operatorname{Var}\left(\hat{y}_{s t}\right)}
$$
where $\operatorname{Var}\left(\hat{y}_{S R S}\right)$ is the variance of the estimate using the SRS estimator, obtained by setting $H=1$ in 231 Eqs. (1) and (2). When $H=1$, then $n_{1}=n$ and $W=w_{1}=1$, where $n_{1}$ and $w_{1}$ are the sample size and 
232 weight for stratum 1, respectively. We note that relative efficiency is defined as the ratio of the 233 variances, but here we estimate it by the ratio of the estimated variances. In general, the greater the 234 value of ERE, the more effective the stratification map at increasing the precision of the parameter 235 estimate.

\section{$236 \quad 3.3$ Analysis}

237 The chief response in this study is ERE for each of seven forest population parameters. Four parameters 238 were status parameters (forest area, forest carbon, forest cutting area, forest planting area) and three 239 were change parameters (forest growth, removals, and mortality). The parameters are based on 240 observed areal extent and individual tree measurements, where trees are defined as having a diameter 241 at breast height of at least $12.7 \mathrm{~cm}$ (5 in). There were $n=977$ FIA plots (forest and non-forest) in the 242 study area (population).

243 For each map of COs (one for each CO type as defined in Section 3.1.2), we used a training sample of 24410,000 pixels to generate solution sets ranging from 4 clusters to 7 clusters. We chose the training 245 sample at random across the scene, using the same training sample for each CO type to control the 246 results for training variation. Making nearest-neighbor assignments for each $\mathrm{CO}$ and solution set yielded 24712 single-layer strata maps: one for each CO type/solution set combination (Table 1). For each of the 12 248 combinations, we assigned to every FIA plot the stratum value for the pixel containing the center of the 249 plot. For each such assignment and parameter of interest we used the PS estimators and the SRS 250 estimators described in section 3.2 to calculate ERE (Eq. 3).

Table 1. Experimental factors in the study.

\begin{tabular}{l|l} 
Factor & Levels \\
\hline Cluster Object Type & Post-Minimum \\
$2002-2009$ \\
Modified 2002-2009 (using \\
& squared NDVI) \\
\hline Number of Strata & $4,5,6,7$
\end{tabular}


255 The main thrust of this research was to test the effectiveness of using a Landsat-derived time series in

256 PS estimation of forest area, total carbon stock, area of planting, area of cutting, total growth, total

257 mortality, and total removals. To accomplish this we quantified the effectiveness by examining ERE

$258\left(\operatorname{Var}\left(\hat{y}_{S R S}\right) / \operatorname{Var}\left(\hat{y}_{S t}\right)\right)$ for each parameter. We note that the FIA program does not typically use SRS

259 estimators but much of the previous research (e.g., McRoberts et al., 2006) has also used SRS estimators

260 to benchmark increase in precision of proposed PS approaches using ERE. Our results should be

261 interpreted in the context of previous research and not be taken as a direct comparison to current FIA

262 PS approaches.

263 Fig. 6 presents the results of the study, with EREs arranged horizontally within the parameter columns to 264 show the effect of the stratum coarseness on ERE. The majority of the EREs were close to 1, with the 265 vast majority of the EREs being within 0.1 of 1 . However, there were a number of cases where the ERE 266 was greater, on the order of 1.2. Recall that an ERE of 1.2 indicates that a sample size of $120 \%$ of the original sample would have been required to achieve the same precision when using SRS estimators. In the case of the status parameters, namely forest area, ERE values approached 2.2. These relatively greater values were less than but comparable with the EREs for status parameters from McRoberts et al. (2006), which had EREs (from other regions of the USA) on the order of 2 or greater. Generally, the finer cluster maps produced larger EREs, which suggests that more partitioning typically allows within-strata variation to be smaller and thus increases the effectiveness of the PS approach. However, the change in

273 ERE from increasing the number of strata was generally quite small, with the exception of the values 274 corresponding to forest area. This dampening may be a result of using the HCA process to define the 275 stratifications, since most stratum assignments from one step to the next remain the same.

All three of the CO types were comparable in terms of their effect on ERE for change parameters, with each CO type associated with the greatest ERE for a different change parameter. There is some

278 intuitive, albeit speculative, reasoning behind these change ERE results. For example, the CO type based on the squared NDVI, here used as a proxy for fractional vegetation cover, yielded the greatest EREs for total removals. Similarly, the 2002-2009 CO type, chosen for its temporal correspondence with the dimensional measurements taken by the FIA crews, yielded the greatest EREs for total growth. the study area was dominated by loblolly pine plantations which, on a stand level, exhibit removal far more routinely than mortality. We hypothesize that after a removal event (e.g., clearcut harvest), the 
natural regeneration or rapid replanting resulted in a reduced impact on our annual NDVI time series.

286 However, in a large mortality event without removal, the canopy becomes dominated by dead crowns, at which point the satellite-based sensor registers a prolonged reduction in NDVI, long enough to be observed at the annual time scale. Further assessment would be needed to verify this hypothesis.

There are several straightforward avenues which could improve subsequent analyses. For example, increasing the overall area of the region of interest would have the effect of incorporating more FIA plots, allowing potentially more strata (due to the higher likelihood of the minimum stratum population

292 being achieved) against the tradeoff of requiring more strata in order to account for the potential

293 increased forest diversity in the area of interest. Further, prior research suggests that more than six 294 strata may yield minimal benefits (Cochran 1977). We could also account for mixed plots more robustly by taking a mode stratum value in a larger spatial neighborhood around each plot center point. Other avenues for improvement will be more challenging to implement. We suspect that a chief contributor to noise in our approach is the temporal disagreement of the CO timeframes with the actual dates of the plot measurements. As an illustrative hypothetical example, a plot which was measured in 2004 and 2011 might have grown undisturbed from 2002 to 2009 but have been harvested in 2010. In this case, the plot would be assigned to a stratum consistent with the undisturbed growth while the measurements show removal. Cases like this contribute to an increase in within-stratum variance, resulting in a lower ERE. The issue is compounded in the post-minimum CO type, where the FIA measurements were generally not from the period used for the COs. Additionally, since sufficient postdisturbance data are needed to generate the $\mathrm{CO}$, plots undergoing recent disturbances cannot easily be included in the stratification for the post-minimum objects. In any case, the PS approach presented here can only benefit from trajectories that more closely tie into the FIA measurement dates. However, since the FIA plots are measured on a rotating panel, such a matching would require a tailoring of Landsat time series to the measurement dates for each plot.

The rate of regrowth after a heavy removal event, as observed in the post-minimum CO trajectory, might be related to the site quality, with higher quality sites displaying a greater regrowth rate. By acting as a proxy for the site index (please see Section 3.1.2.1), the strata maps produced by the postminimum object type might also be employed anywhere that site index is, in wall to wall raster format. This possibility is an area worth exploring, but it would require more precise estimates of the time and severity of disturbances, attainable via any of the multitemporal change detection methods available today (Brooks et al., 2014; Huang et al., 2010; Kennedy et al., 2010; Zhu et al., 2012). 
Harmonic coefficient estimates from a Landsat time series improved the precision of change parameter

317 estimates via a stratification that generated maps related to land cover. This is encouraging given the recent emergence of harmonic regression in the literature as a means to fit Landsat time series data for subsequent change analysis (Brooks et al., 2012; Zhu et al. 2012; Zhu et al., 2015). There is potential to use such coefficients for more general land cover/land use classification, as bases for change detection,

321 or as predictors in models relating images to plot measurements. This potential is a chief focus for 322 related future work.

323 In this study, we used PS estimation, but the data used to develop the strata maps could also be used in 324 a model-assisted estimation framework (Särndal et al., 1992; McRoberts, 2010). One potential approach 325 is using NDVI trajectory as a proxy or model of change. In simple terms, the relationship between fieldobserved values and the model of change would form the basis of the estimator. Model-assisted estimation of forest change parameters remains a fruitful area of research.

This study focused on improving precision for forest change parameter estimates by using trajectorybased stratum assignments. We used several variations on this general approach to explore its potential with no clear expectations of which variation would be optimal. In general, the EREs from this research suggest modest increases in precision as compared to the SRS variance estimates. Even so, the PS estimates based on our stratification maps had smaller estimated variances for growth, mortality, and removal, offering evidence that multitemporal stratification methods incorporating fractional vegetation cover and post-disturbance recovery can improve the precision of change parameter estimates.

\section{Acknowledgments}

337 We want to thank the USGS for making the Landsat archive freely available, enabling this study. We would also like to thank Karl Sorensen for his work in preprocessing the Landsat data through LEDAPS, as well as the reviewers for their positive and helpful feedback. This work was supported by the USDA Forest Service Cooperative Agreement with Virginia Tech (Grant No. 10-CA-11330145-158). It was also

341 supported by the Landsat Science Team (USGS contract number G12PC00073 and related contracts), the 342 Pine Integrated Network: Education, Mitigation, and Adaptation Project (PINEMAP, Coordinated

343 Agricultural Project funded in 2011 by the USDA National Institute of Food and Agriculture), the 344 McIntire-Stennis Cooperative Forestry Research program (USDA CSREES, Project No. VA-1007054), and 345 the Department of Forest Resources and Environmental Conservation at Virginia Tech. 


\section{References}

Bechtold, W. A. and Scott, C. T. (2005) "The forest inventory and analysis plot design." Gen. Tech. Rep. SRS-80. Asheville, NC: U.S. Department of Agriculture, Forest Service, Southern Research Station, 37-52.

Bickford, C. A. (1952). "The sampling design used in the forest survey of the Northeast." Journal of Forestry, 50(4), 290-293.

Brooks, E. B., Thomas, V. A., Wynne, R. H., and Coulston, J. W. (2012) "Fitting the multitemporal curve: a Fourier series approach to the missing data problem in remote sensing analysis." IEEE Transactions on Geosciences and Remote Sensing, 50(9), 3340-3353.

Brooks, E. B., Wynne, R. H., Thomas, V. A., Blinn, C. E., and Coulston, J. W. (2014) “On-the-fly massively multitemporal change detection using statistical quality control charts and Landsat data." IEEE Transactions on Geosciences and Remote Sensing, 52(6), 3316-3332.

Burkman, B. (2005a) "Forest Inventory and Analysis sampling and plot design." FIA Fact Sheet Series. http://www.fia.fs.fed.us

Burkman, B. (2005b) "Forest Inventory and Analysis data collection and analysis." FIA Fact Sheet Series. http://www.fia.fs.fed.us

Chavez, P. S. (1988). “An improved dark-object subtraction technique for atmospheric scattering correction of multispectral data." Remote Sensing of Environment, 24(3), 459-479.

Chojnacky, D. C. (1998) Double sampling for stratification: a forest inventory application in the Interior West. US Department of Agriculture, Forest Service, Rocky Mountain Research Station, 1998.11

Choudhury, B. J., Ahmed, N. A., Idso, S. B., Reginato, R. J., and Daughtry, C. S. T. (1994) "Relations between evaporation coefficients and vegetation indices studied by model simulations." Remote Sensing of Environment, 50(1), 1-17.

Cochran, W. G. (1977) Sampling Techniques. $3^{\text {rd }}$ edition. Wiley, New York. p.134-135.

Fransson, J. E. S. (2000) “Estimation of forest parameters using CARABAS-II VHF SAR data." IEEE Transactions on Geosciences and Remote Sensing, 38(2), 720-727.

Fry, J., Xian, G., Jin, S., Dewitz, J., Homer, C., Yang, L., Barnes, C., Herold, N., and Wickham, J., 2011. "Completion of the 2006 National Land Cover Database for the Conterminous United States." Photogrammetric Engineering \& Remote Sensing, 77(9), 858-864.

Hansen, M.H., and Wendt, D.G. (2000). "Using classified Landsat Thematic Mapper data for stratification in a statewide forest inventory." Proceedings of the First Annual Forest Inventory and Analysis Symposium (November 1999), 20-27. 
Hansen, M. C., Stehman, S. V., Potapov, P. V., Arunarwati, B., Stolle, F., and Pittman, K. (2009) "Quantifying changes in the rates of forest clearing in Indonesia from 1990 to 2005 using remotely sensed data sets." Environmental Research Letters, 4(3).

Hoppus, M. L., and Lister, A. J. (2003) “A statistically valid method for using FIA plots to guide spectral class rejection in producing stratification maps." Proceedings of the Third Annual Forest Inventory and Analysis Symposium (October 2001), 17-19.

Huang, C., Goward, S., Masek, J., Thomas, N., Zhu, Z., and Vogelmann, J. (2010) “An automated approach for reconstructing recent forest disturbance history using dense Landsat time series stacks." Remote Sensing of Environment, 114(1), 183-198.

Katila, M., and Tomppo, E. (2002) "Stratification by ancillary data in multisource forest inventories employing k-nearest-neighbour estimation." Canadian Journal of Forest Research, 32(9), 15481561.

Kennedy, R., Yang, Z., and Cohen, W. (2010) "Detecting trends in forest disturbance and recovery using yearly Landsat time series:1. LandTrendr - temporal segmentation algorithms." Remote Sensing of Environment, 114(12), 2897-2910.

LaBau, V. J. (2007). A history of the forest survey in the United States: 1830-2004. US Dept. of Agriculture, Forest Service FS-877.

Masek, J. G., Vermote, E. F., Saleous, N. E., Wolfe, R., Hall, F. G., Huemmrich, K. F., Gao, F., Kutler, J., and Lim, T.-K. (2006) "A Landsat surface reflectance dataset for North America, 1990-2000." IEEE Geoscience and Remote Sensing Letters, 3(1), 68-72.

McRoberts, R. E., and Hansen, M. H. (1999) “Annual forest inventories for the North Central region of the United States." Journal of Agricultural, Biological, and Environmental Statistics, 4(4), 361-371.

McRoberts, R. E., Wendt, D. G., Nelson, M. D., and Hansen, M. H. (2002a) “Using a land cover classification based on satellite imagery to improve the precision of forest inventory area estimates." Remote Sensing of Environment, 81(1), 36-44.

McRoberts, R. E., Nelson, M. D., and Wendt, D. G. (2002b) "Stratified estimation of forest area using satellite imagery, inventory data, and the k-Nearest Neighbors technique." Remote Sensing of Environment, 81(2-3), 457-468.

McRoberts, R. E., Holden, G. R., Nelson, M. D., Liknes, G. C., and Gormanson, D. D. (2006) “Using satellite imagery as ancillary data for increasing the precision of estimates for the Forest Inventory and Analysis program of the USDA Forest Service." Canadian Journal of Forest Research, 36, 29682980. 
McRoberts, R.E. (2010). "Probability-based and model-based approaches to inference using satellite imagery as ancillary data." Remote Sensing of Environment, 114, 1017-1025.

McRoberts, R. E., Gobakken, T., and Naesset, E. (2012) "Post-stratified estimation of forest area and growing stock volume using lidar-based stratifications." Remote Sensing of Environment, 125, 157166.

Moody, A. and Johnson, D. M. (2001) "Land-surface phenologies from AVHRR using the discrete Fourier transform," Remote Sensing of Environment, 75(3), 305-323.

Musy, R. F., Wynne, R. H., Blinn, C. E., Scrivani, J. A., and McRoberts, R. E. (2006) “Automated forest area estimation using iterative guided spectral class rejection." Photogrammetric Engineering and Remote Sensing, 72(8), 949-960.

Nilsson, M., Holm, S., Reese, H., Wallerman, J., and Engberg, J. (2005) “Improved forest statistics from the Swedish National Forest Inventory by combining field data and optical satellite data using poststratification." Proceedings of ForestSAT, 31, 22-26.

Patterson, P. L., Coulston, J. W., Roesch, F. A., Westfall, J. A., and Hill, A. D. (2012) “A primer for nonresponse in the US forest inventory and analysis program." Environmental Monitoring and Assessment, 184(3), 1423-1433.

Roerink, G. J., Menenti, M., Verhoef, W. (2000) “Reconstructing cloud-free NDVI composites using Fourier analysis of time series," International Journal of Remote Sensing, 21(9), 1911-1917.

Sampson, D. A., Wynne, R. H., and Seiler, J. (2008) “Edaphic and climatic effects on forest stand development, net primary production, and net ecosystem productivity simulated for Coastal Plain loblolly pine in Virginia." Journal of Geophysical Research, 113(G1).

Särndal, C.-E., Swensson, B., \& Wretman, J. (1992) Model assisted survey sampling. Springer-Verlag, Inc. New York. 694 p.

Scott, C. T., Bechtold, W. A., Reams, G. A., Smith, W. D., Westfall, J. A., Hansen, M. H., and Moisen, G. G. (2005) "Sample-based estimators used by the Forest Inventory and Analysis national information management system." The Enhanced Forest Inventory and Analysis Program - National Sampling Design and Estimation Procedures. U.S. Forest Service General Technical Report, SRS-80. pp. 43-67.

Tomppo, E., Olsson, H., Stahl, G., Nilsson, M., Hagner, O., and Katila, M. (2008) “Combining national forest inventory field plots and remote sensing data for forest databases." Remote Sensing of Environment, 112(5), 1982-1999.

Tucker, C. J. (1979) "Red and photographic infrared linear combinations for monitoring vegetation." Remote Sensing of Environment, 8(2), 127-150. 
442 USGS Global Visualization Viewer (GLOVIS) website. http://glovis.usgs.gov/

443 Ward, J. H. Jr., 1963. "Hierarchical grouping to optimize an objective function." Journal of the American $444 \quad$ Statistical Association, 58(301), 236-244.

445 Wayman, J. P., Wynne, R. H., Scrivani, J. A., and Reams, G. A. (2001) “Landsat TM-based forest area 446 estimation using iterative guided spectral class rejection." Photogrammetric Engineering and 447 Remote Sensing, 67(10), 1155-1166.

448 Westfall, J. A., Patterson, P. L., and Coulston, J. W. (2011) "Post-stratified estimation: within-strata and 449 total sample size recommendations." Canadian Journal of Forest Research, 41(5), 1130-1139.

450 Wynne, R.H., Oderwald, R. G., Reams, G.A., and Scrivani, J.A. (2000) “Optical remote sensing for forest 451 area estimation." Journal of Forestry, 98(5), 31-36.

452 Zhu, Z., Woodcock, C. E., and Olofsson, P. (2012) “Continuous monitoring of forest disturbance using all 453 available Landsat imagery." Remote Sensing of Environment, Landsat Legacy Special Issue, 122, 7545491

455 Zhu, Z., Woodcock, C. E., Holden, C., and Yang, Z. (2015) “Generating synthetic Landsat images based on 456 all available Landsat data: Predicting Landsat surface reflectance at any given time." Remote 457 Sensing of Environment, 162, 67-83.

458 
460 Figure 1. Study area. Inset RGB are the mean normalized difference vegetation index (NDVI) values for 461 3-year groups centered on 1985, 1988, and 1991, respectively.

462 Figure 2. Temporal distribution of Landsat Thematic Mapper (TM) images.

463 Figure 3. Running mean method on NDVI values, detailed for three example pixels from different loblolly 464 pine (Pinus taeda) stands. It is evident from the time series trajectories that pixels $A$ and $C$ underwent a 465 harvest of some degree, although the degree and timing of each harvest varied. Pixel $B$ appears to have 466 suffered a decrease in vegetation in the late 1990s as well.

467 Figure 4. Clustering objects (COs) computed from the example pixels of Figure 3. Note how the two 468 most similar objects vary according to which CO type is chosen.

469 Figure 5. HCA algorithm, demonstrated on a sample of trajectories for three clusters (colors). a) From 470 the full population, take a random sample (sample in black). b) Classify this sample according to self471 similarity, and compute cluster centroids (dashed lines). c, d) In the original population, classify each 472 element based on the nearest centroid.

473 Figure 6. Estimated relative efficiency (ERE) values for the post-stratified estimator for the total for each 474 of the forest parameters assessed in this study. The relative sizes of the plot elements describe the 475 coarseness of the strata, with larger elements to the left of each column corresponding to solution sets 476 with fewer strata. 
Temporal Distribution of Landsat Images

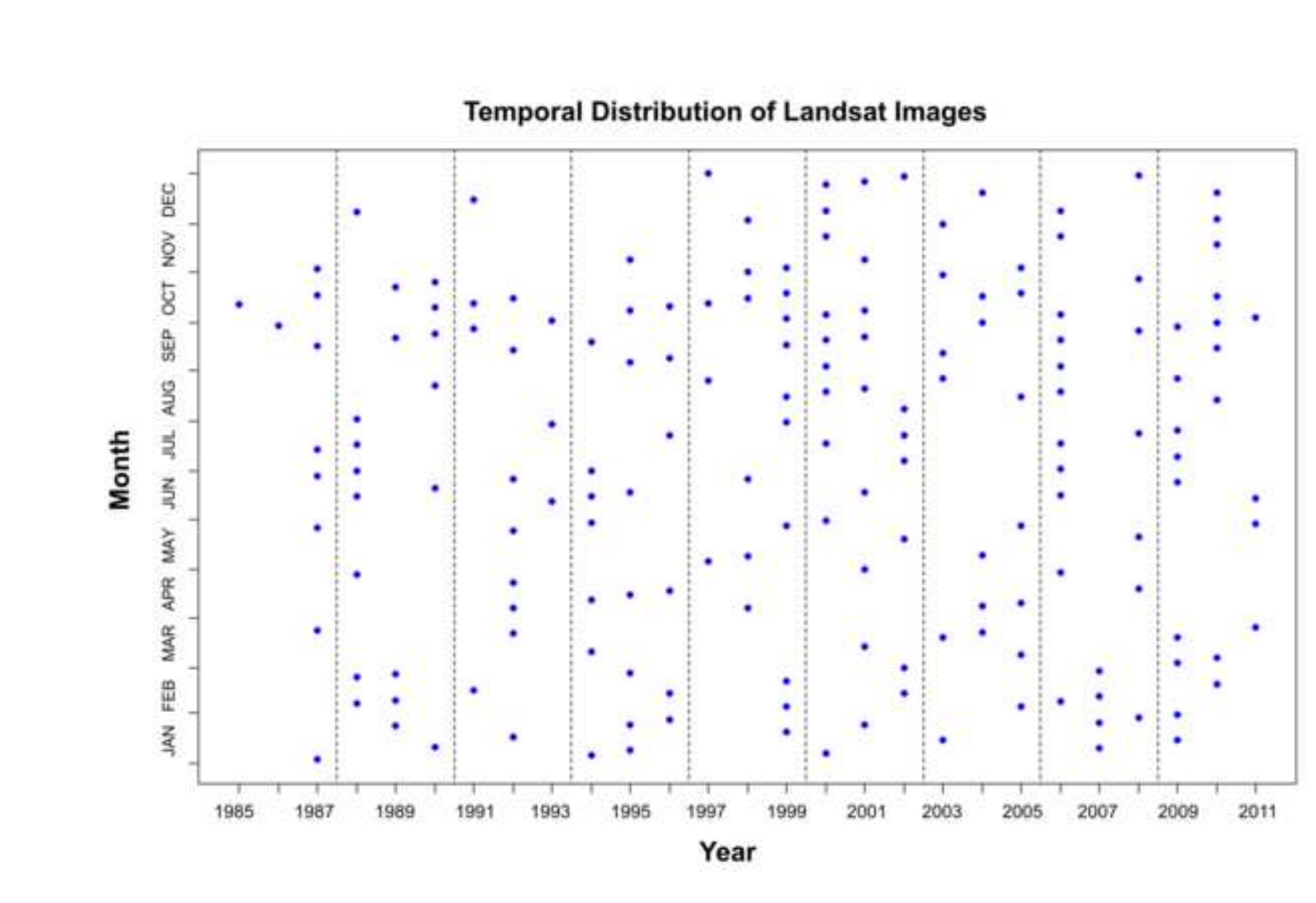

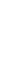

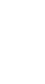

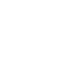

$\sqrt{2}$

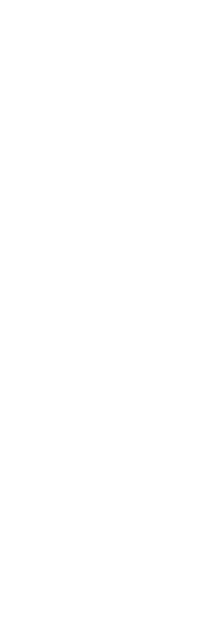

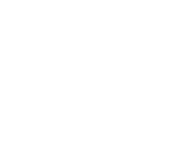
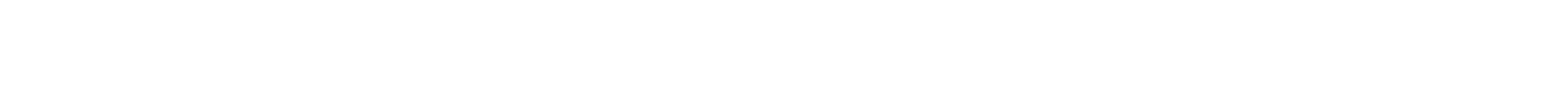

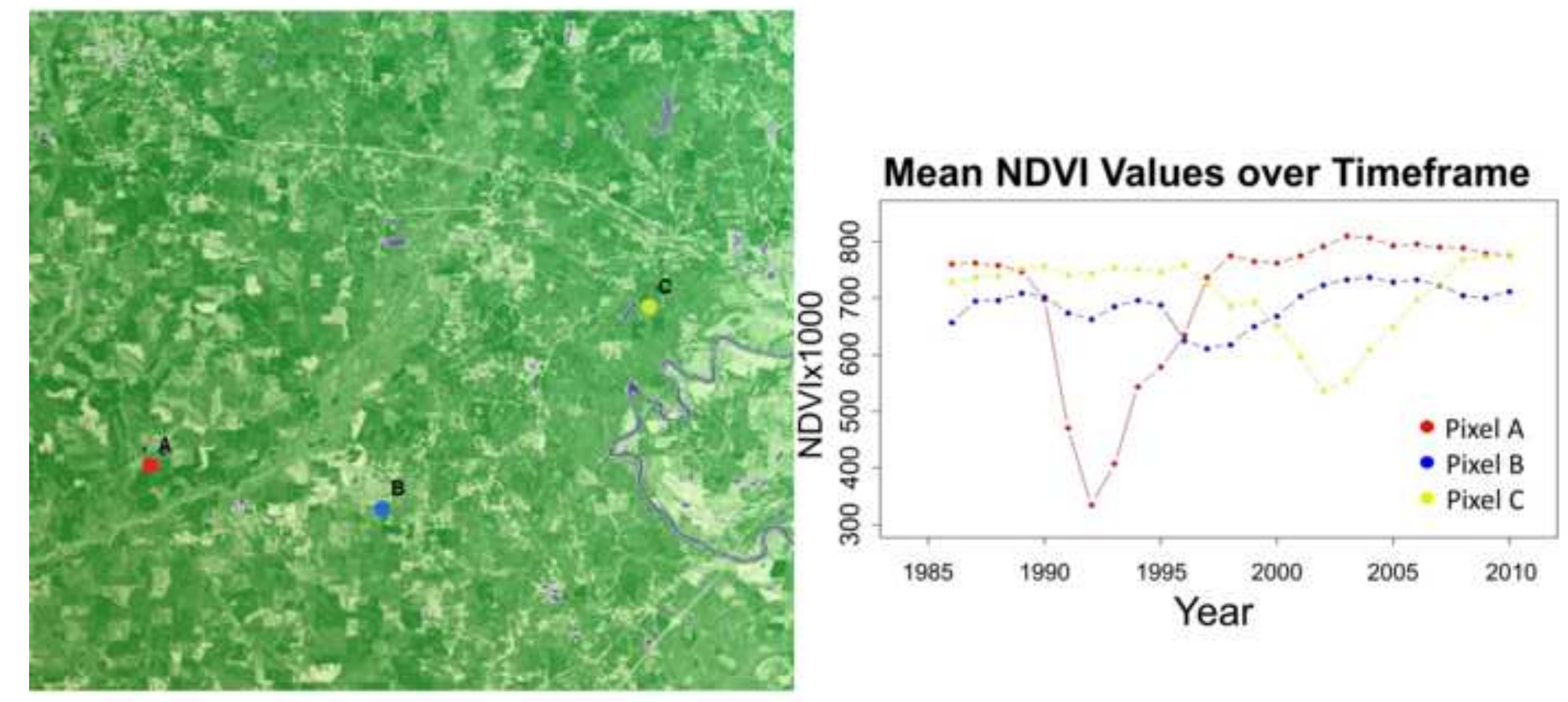


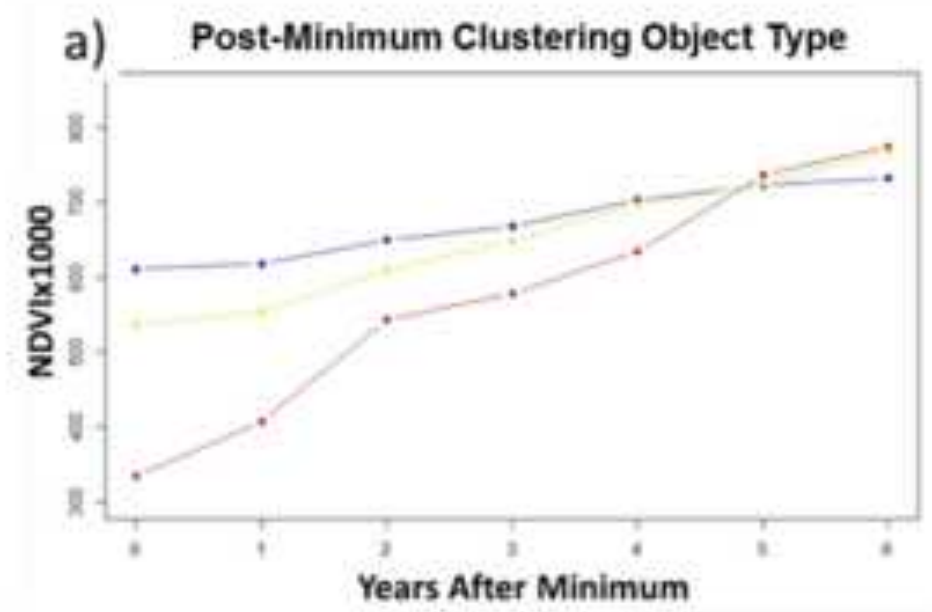

- Pixel A

- Pixel B

- Pixel C
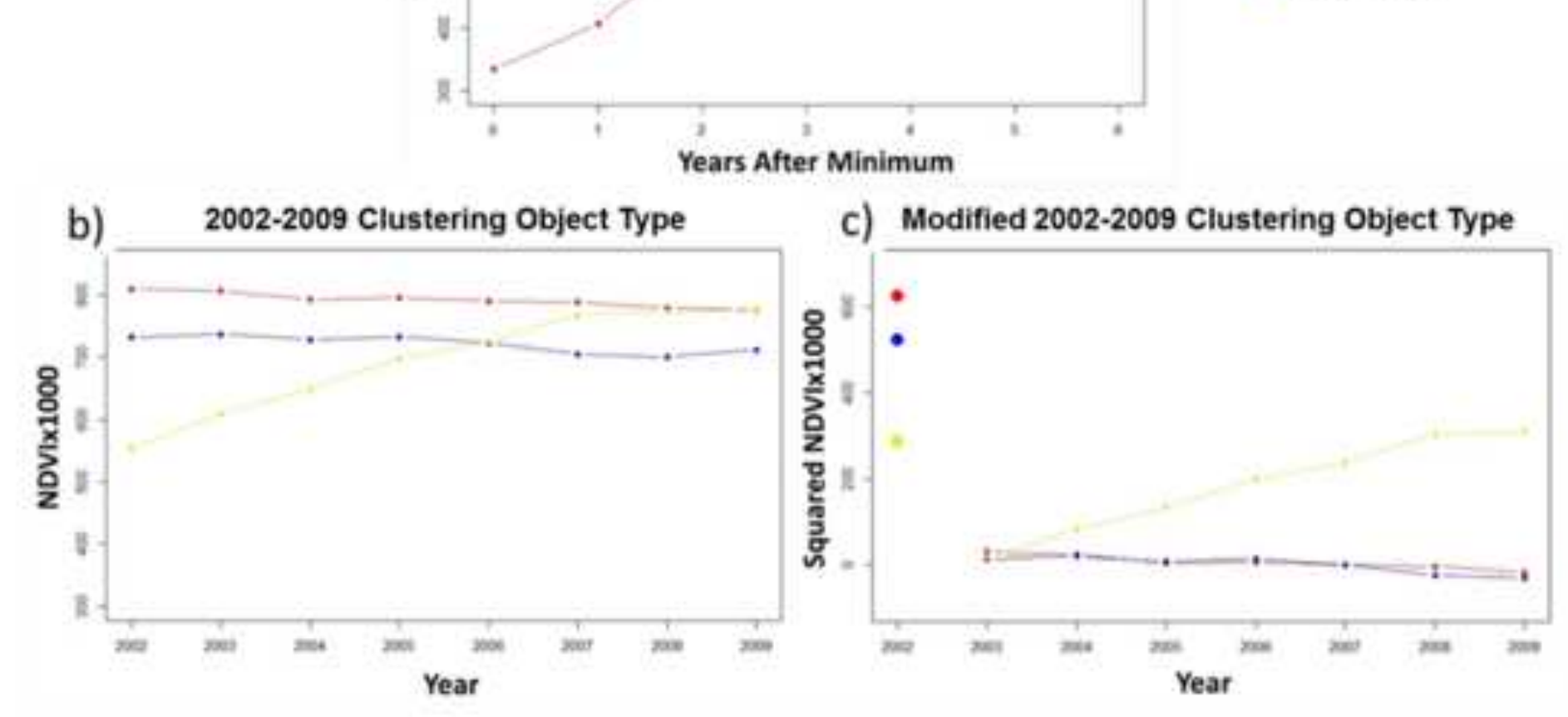
Estimated Relative Efficiency for all Clustering Object Types and Parameters

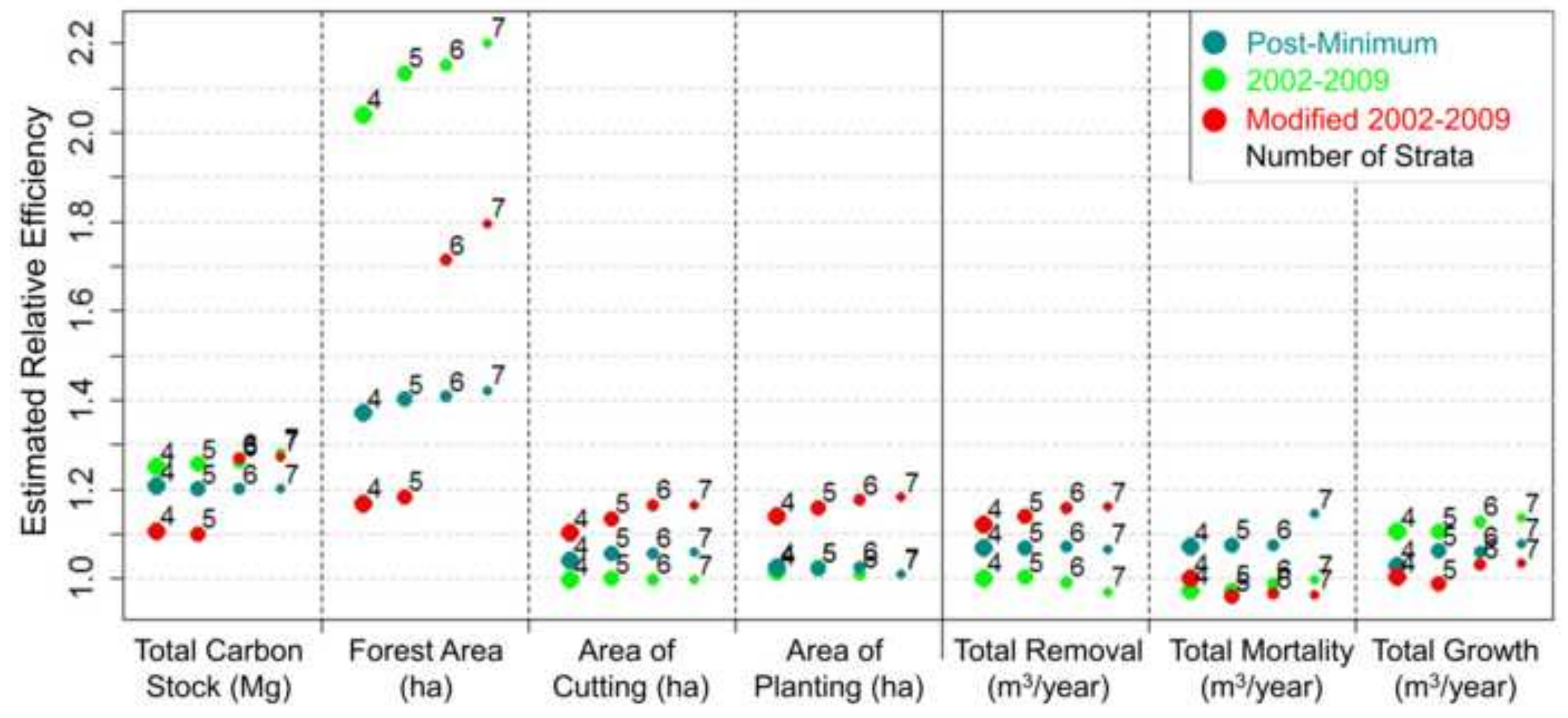

